\title{
KD-II 型碳化硅纤维热输运性质的实验研究
}

\author{
施徐国 ${ }^{1}$, 李明远 ${ }^{2}$, 马维刚 ${ }^{1}$, 周新贵 ${ }^{2}$, 张 兴 $^{1}$
}

(1. 清华大学工程力学系, 热科学与动力工程教育部重点实验室, 北京 $100084 ; 2$. 国防科技大学 新型陶瓷纤维 及其复合材料重点实验室, 长沙 410073)

摘 要: $\mathrm{SiC}$ 纤维是复合材料 $\mathrm{SiC}_{\mathrm{f}} / \mathrm{SiC}$ 中的组分材料, 其力学性能已经得到实验验证, 但热学性能尚未见报道。本研 究采用综合 $\mathrm{T}$ 型法测量了不同温度热处理的 $\mathrm{KD}-\mathrm{II}$ 型 $\mathrm{SiC}$ 纤维在 80 300 K 温度范围内的电导率、热导率和塞贝克 系数, 热处理温度分别为 $1400 、 1500$ 和 $1600^{\circ} \mathrm{C}$ 。研究发现, 在实验设定的热处理温度范围内, $\mathrm{SiC}$ 纤维电导率不随 热处理温度改变而改变, 但其热导率随热处理温度升高有显著的变化。在环境温度为 $290 \mathrm{~K}$ 时, $1600^{\circ} \mathrm{C}$ 热处理的 $\mathrm{SiC}$ 纤维热导率为 $11.6 \mathrm{~W} \cdot \mathrm{m}^{-1} \cdot \mathrm{K}^{-1}$, 比未热处理的材料提高了 $42 \%$ 以上。

关 键 词: 碳化硅纤维; 热导率; 电导率

中图分类号: O482 文献标识码: A

\section{Experimental Study on Thermal Transport Property of KD-II SiC Fiber}

\author{
SHI Xu-Guo ${ }^{1}$, LI Ming-Yuan ${ }^{2}$, MA Wei-Gang ${ }^{1}$, ZHOU Xin-Gui ${ }^{2}$, ZHANG Xing $^{1}$
}

(1. Key Laboratory for Thermal Science and Power Engineering of Ministry of Education, Department of Engineering Mechanics, Tsinghua University, Beijing 100864, China; 2. Science and Technology on Advanced Ceramic Fiber and Composites Laboratory, National University of Defense Technology, Changsha 410073, China)

Abstract: $\mathrm{SiC}$ fiber is a component material of $\mathrm{SiC}_{\mathrm{f}} / \mathrm{SiC}$, its mechanical properties have been verified experimentally. In present work, the electrical conductivity, thermal conductivity and Seebeck coefficient of KD-II SiC fibers heat-treated at different temperatures were measured from $80 \mathrm{~K}$ to $300 \mathrm{~K}$ by a comprehensive T-type method. Heat treatment temperatures were $1400^{\circ} \mathrm{C}, 1500^{\circ} \mathrm{C}$ and $1600^{\circ} \mathrm{C}$, respectively. It is found that the electrical conductivity of $\mathrm{SiC}$ fiber does not change with the heat treatment temperature, however the thermal conductivity of SiC fiber is significantly enhanced as the heat treatment temperature increases. At the environment temperature of $290 \mathrm{~K}$, the thermal conductivity of $\mathrm{SiC}$ fiber after heat-treated at $1600^{\circ} \mathrm{C}$ is $11.6 \mathrm{~W} \cdot \mathrm{m}^{-1} \cdot \mathrm{K}^{-1}$, which is $42 \%$ higher than that of unheated $\mathrm{SiC}$ fiber.

Key words: $\mathrm{SiC}$ fiber; thermal conductivity; electrical conductivity

连续 $\mathrm{SiC}$ 纤维增强碳化硅基陶瓷复合材料 $\left(\mathrm{SiC}_{\mathrm{f}} / \mathrm{SiC}\right)$ 是一种具有良好力学性能和抗氧化性能 的陶瓷基复合材料, 在核防护材料中具有重要的潜 在应用价值。热导率对 $\mathrm{SiC}_{\mathrm{f}} / \mathrm{SiC}$ 复合材料的应用有 着重要的影响。 $\mathrm{SiC}_{\mathrm{f}} / \mathrm{SiC}$ 复合材料作为结构材料和 功能材料时, 对其热导率的要求有所不同 ${ }^{[1]}$ : 结构
材料面临高热流传热的问题, 要求材料热导率尽可 能高; 而起热绝缘作用的插件(功能材料)则要求材 料热导率尽可能低。

目前，商用复合材料在热导率方面存在不足 ${ }^{[2]}$, 面 向热导率约为 $15 \mathrm{~W} \cdot \mathrm{m}^{-1} \cdot \mathrm{K}^{-1}$, 法向热导率为 $9 \mathrm{~W} \cdot \mathrm{m}^{-1} \cdot \mathrm{K}^{-1[3]}$, 在高温和辐照条件下复合材料的法向热导率则更低,

收稿日期：2017-09-02; 收到修改稿日期：2017-10-19

基金项目: 国家自然科学基金重点项目(51327001) National Natural Science Foundation of China(51327001)

作者简介: 施徐国(1990-), 男, 博士研究生. E-mail: sxg12@mails.tsinghua.edu.cn

通讯作者: 张 兴, 教授. E-mail: x-zhang@tsinghua.edu.cn 
与工程应用要求 $\left(20 \mathrm{~W} \cdot \mathrm{m}^{-1} \cdot \mathrm{K}^{-1}\right)$ 还有一定的差距。因 此, 高热导率 $\mathrm{SiC}_{\mathrm{f}} / \mathrm{SiC}$ 材料的开发已成为国内外的 研究热点。 2002 年, Jones 等 ${ }^{[4]}$ 提出了 $\mathrm{SiC}_{\mathrm{f}} / \mathrm{SiC}$ 复合 材料热导率的 Hasselman-Johnson 估算模型，模型中 指出提高 $\mathrm{SiC}_{\mathrm{f}} / \mathrm{SiC}$ 复合材料热导率的三个途径: 提 高基体热导率、提高纤维热导率和降低接触热阻。 为了提高 $\mathrm{SiC}_{\mathrm{f}} / \mathrm{SiC}$ 复合材料热导率, 需要深入理解 $\mathrm{SiC}$ 纤维热导率随热处理温度的变化规律。

1978 年, Yajima 等 ${ }^{[5]}$ 提出了以聚碳硅烷(Polycarbosilane, PCS)为先驱体制备碳化硅纤维的方法, 先驱体转化法可以分为先驱体合成、先驱体熔融、 纺丝和高温烧结四个步骤。先驱体制备碳化硅纤维 历经了三代 ${ }^{[6]}$ 。第一代碳化硅纤维的特点是成分中包 含多余的碳和氧, 多余的氧形成了无定型的 Si-C-O 晶 间相, $\beta-\mathrm{SiC}$ 晶粒相的比例小, 发生氧化时, 其强度 显著降低 ${ }^{[7]}$ 。Nippon Carbon 公司生产的 Nicalon 和 Ube Industries 生产的 Tyranno Lox-M 纤维是第一代 碳化硅纤维的代表。第二代碳化硅纤维的特点是氧 含量低, $\beta$ - $\mathrm{SiC}$ 晶粒尺寸较大。与第一代纤维类似, 第二代碳化硅纤维依然呈无定型态。Nippon Carbon 公司生产的 Hi-Nicalon 和 Ube Industries 生产的 Tyranno Lox-E 纤维是第二代碳化硅纤维的代表。第 三代碳化硅纤维的特点是近理想化学计量比, 进一 步减少了纤维中的单相碳和氧。第三代碳化硅纤维的 $\beta-\mathrm{SiC}$ 晶粒尺寸更大, 约 50 200 $\mathrm{nm}^{[8]}$ 。Nippon Carbon 公司生产的 Hi-Nicalon S 和 Ube Industries 生产的 Tyranno SA 纤维是第三代碳化硅纤维的代表。

$\mathrm{KD}$ 系列 $\mathrm{SiC}$ 纤维是由国防科技大学于 1985 年 开始主导研究的系列 $\mathrm{SiC}$ 纤维, 目前已包含 KD-I 型、 KD-II 型和 KD-S 型 $\mathrm{SiC}$ 纤维。其中 $\mathrm{KD}-\mathrm{II}$ 型纤 维的拉伸强度达 $3.0 \mathrm{GPa}$, 拉伸模量达 $278 \mathrm{GPa}^{[9]}$, 达到日本同类产品的指标。但上述材料的典型热学 特性(热导率)尚未研究。针对国防科技大学研制的 $\mathrm{KD}-\mathrm{II}$ 型 $\mathrm{SiC}$ 纤维, 本工作测量其经 $1400^{\circ} \mathrm{C} 、 1500^{\circ} \mathrm{C}$ 和 $1600^{\circ} \mathrm{C}$ 热处理后纤维的电导率、热导率和塞贝克 系数。

\section{1 实验测量原理}

$\mathrm{SiC}$ 纤维的电导率、热导率和塞贝克系数用综 合 $\mathrm{T}$ 型法测量。综合 $\mathrm{T}$ 型法中的待测线以 $\mathrm{T}$ 型搭接 与热线传感器相连, 如图 1 所示。 $\mathrm{T}$ 型搭接包含以 下特点: 在基底上有 6 个与基底绝缘且彼此绝缘的电 极, 这些电极与基底导热良好且热容远大于热线和 待测线，因此可以看作温度等于环境温度的热沉。 以铂为材料的热线横跨在 4 个电极间, 悬浮于基底

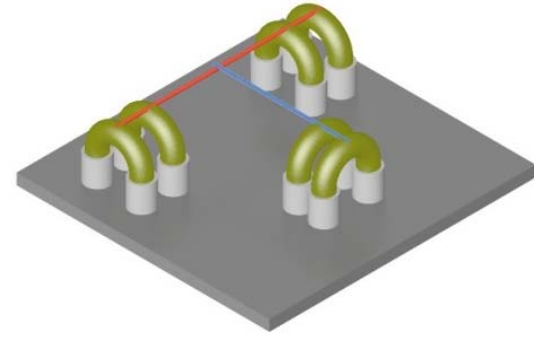

图 1 T 型搭接示意图

Fig. 1 Schematic diagram of T-type assembling

之上; 待测线一端与两个电极相连, 一端搭接与热 线中点位置。在热线左侧的两个电极称为热线的正 极，而热线右侧的两个电极称为热线的负极。 $\mathrm{T}$ 型 搭接是四线制 $\mathrm{T}$ 型法、直流 $\mathrm{T}$ 型法和赛贝克 $\mathrm{T}$ 型法 的基础, 实现了对同一个样品一次搭接无需转移即 可实现综合测量。

（1）四线制 $\mathrm{T}$ 型法，即在 $\mathrm{T}$ 型搭接的基础上，使 用四线制测量待测线的电阻，再结合待测线的尺寸， 计算待测线的电导率。待测线电阻 $R_{\mathrm{f}}$ 可以表示为测 量的电压和电流线性拟合的斜率 $R_{\mathrm{f}}=\operatorname{slop}(I, U)$, 待 测线电导率 $\sigma_{\mathrm{f}}$ 可以表示为 $\sigma_{\mathrm{f}}=l_{\mathrm{f}} /\left(A_{\mathrm{f}} R_{\mathrm{f}}\right)$ 。其中 $l_{\mathrm{f}}$ 表示 $\mathrm{SiC}$ 纤维的长度, $A_{\mathrm{f}}$ 表示 $\mathrm{SiC}$ 纤维的截面积。

(2) 直流 T型法, 也称作稳态热线法, 是一种测 量一维材料热导率的电学测量方法。直流 $\mathrm{T}$ 型法最 初由 Zhang 等 ${ }^{[10]}$ 提出, 用于测量单根碳纤维的热导 率。直流 $\mathrm{T}$ 型法包含一根作为传感器的热线和一根 样品线, 它的基本测量过程包括以下 4 步: a) 搭接 样品。将热线与样品 $\mathrm{T}$ 型搭接后放置于气压小于 $10^{-4} \mathrm{~Pa}$ 、温度波动小于 $0.1 \mathrm{~K}$ 的真空恒温槽内; b) 测 量。在热线两端通直流电, 通过测量热线电阻与热线 加热功率的关系，计算热线-待测线系统的总热阻 $\left.k=\operatorname{slop}(P, R) /\left(\beta_{273.15} R_{273.15}\right) ; \mathrm{c}\right)$ 标定。取下待测线 后重复步骤 $\mathrm{b}$, 标定热线系统的热阻 $k_{0}=\operatorname{slop}\left(P_{0}, R_{0}\right)$ / $\left.\left(\beta_{273.15} R_{273.15}\right) ; \mathrm{d}\right)$ 计算测量结果。在准一维有内热 源的稳态导热模型的基础上对比步骤 $\mathrm{b}$ 与步骤 $\mathrm{c}$ 的 结果，计算待测线的热导率。待测线的热导率 $\lambda_{\mathrm{f}}$ 可 以表达为:

$$
\lambda_{\mathrm{f}}=\frac{l_{\mathrm{f}} A_{\mathrm{h}}}{l_{\mathrm{h}} A_{\mathrm{f}}\left(\frac{3}{16} \frac{1}{1-k / k_{0}}-\frac{1}{4}\right)} \lambda_{\mathrm{h}}
$$

其中， $l_{\mathrm{h}}$ 表示热线的长度， $A_{\mathrm{h}}$ 表示热线的截面积， $l_{\mathrm{f}}$ 表示 $\mathrm{SiC}$ 纤维的长度, $A_{\mathrm{f}}$ 表示 $\mathrm{SiC}$ 纤维的截面积。

(3) 交流加热-直流探测 $\mathrm{T}$ 型法是由 Miao 等 ${ }^{[11]}$ 在直流 $\mathrm{T}$ 型法的基础上提出的一种测量待测线塞贝 克系数的方法。交流加热是指在热线两端加载正弦 
交流电 $I_{0} \sin (\omega t)$, 此时在热线温升分布包含一个谐 波分量与一个稳态分量, 可以表示为:

$$
T_{\mathrm{h}}\left(x_{\mathrm{h}}\right)=T_{\mathrm{h}, \text { static }}\left(x_{\mathrm{h}}\right)+T_{\mathrm{h} \text {,harmonic }}\left(x_{\mathrm{h}}, t\right)
$$

同理, 在热线与待测线的搭接点与待测线的热沉 端之间的温升也可以分为谐波分量和稳态分量, 即:

$$
\Delta T_{\mathrm{f}}=\Delta T_{\mathrm{f}, \text { static }}+\Delta T_{\mathrm{f} \text {,harmonic }}(t)
$$

求解热线的一维导热方程, 可以将节点稳态温 升 $\Delta T_{\mathrm{f}, \mathrm{static}}$ 表示为:

$$
\Delta T_{\mathrm{f}, \text { static }}=\frac{I_{0}^{2} R_{\mathrm{h}} l_{\mathrm{h}} l_{\mathrm{f}}}{4\left(A_{\mathrm{f}} \lambda_{\mathrm{f}} l_{\mathrm{h}}+4 A_{\mathrm{h}} \lambda_{\mathrm{h}} l_{\mathrm{f}}\right)}
$$

其中, $l_{\mathrm{h}}$ 表示热线的长度, $A_{\mathrm{h}}$ 表示热线的截面积, $\lambda_{\mathrm{h}}$ 表示热线热导率, $R_{\mathrm{h}}$ 表示热线电阻, $l_{\mathrm{f}}$ 表示 $\mathrm{SiC}$ 纤维的长度, $A_{\mathrm{f}}$ 表示 $\mathrm{SiC}$ 纤维的截面积, $\lambda_{\mathrm{f}}$ 表示 $\mathrm{SiC}$ 纤维热导率。温升的正弦分量引起了待测线两 端塞贝克电势的正弦变化, 而温升的稳态分量引起 了塞贝克电势的直流分量。通过直流电压表检测待 测线两端的直流塞贝克电动势, 可以计算待测线的 塞贝克系数 $S$ 为:

$$
S=-\frac{V_{S}}{\Delta T_{\mathrm{f}, \mathrm{static}}}
$$

本工作采用光学显微镜和电动位移平台测量热 线和样品的长度, 测量精度为 $0.5 \mu \mathrm{m}$, 对于 $30 \mathrm{~mm}$ 长的热线, 其相对不确定度小于 $2 \times 10^{-5}$, 对于 $5 \mathrm{~mm}$ 长的样品, 其相对不确定度小于 $10^{-4}$ 。采用扫描电 子显微镜测量样品直径, 测量精度为 $10 \mathrm{~nm}$, 对于 $12 \mu \mathrm{m}$ 直径的样品, 其相对不确定度小于 $10^{-3}$ 。

根据本研究的测试回路, 四线制 $\mathrm{T}$ 型法测量待 测线电导率的计算公式可以进一步表示为:

$$
\sigma_{\mathrm{f}}=\frac{4 l_{\mathrm{f}} U_{\mathrm{s}}}{\pi D_{\mathrm{f}}^{2} U_{\mathrm{f}} R_{\mathrm{s}}}
$$

其中 $U_{\mathrm{f}}$ 为待测线两端电压, $U_{\mathrm{s}}$ 为标准电阻两端电 压, $l_{\mathrm{f}}$ 为待测线长度, $D_{\mathrm{f}}$ 为待测线直径, $R_{\mathrm{s}}$ 为标准 电阻阻值。本研究使用两台 Keithley 2002 作为电压 表, 其不确定度的表达式为: $\delta U= \pm\left(\alpha \times U_{\mathrm{m}}+\beta \times\right.$ $\left.U_{\mathrm{r}}\right) / 1000000$, 其中 $U_{\mathrm{m}}$ 表示电压测量值, $U_{\mathrm{r}}$ 表示 电压表量程。以典型情况为例, 电压表量程选取为 $200 \mathrm{mV}$, 实测电压值为 $100 \mathrm{mV}$, 此时电压表不确定 度为 $\delta U=0.0043 \mathrm{mV}$, 相对不确定度小于 $5 \times 10^{-5}$ 。 标准电阻型号为 YOKOYAWA 2792, 其相对不确定 度小于 $3 \times 10^{-3}$ 。根据电导率误差传递公式, 测量 $\mathrm{SiC}$ 纤维电导率的相对不确定度小于 $0.2 \%$ 。

直流 $\mathrm{T}$ 型法中测量系统的总热阻的表达式为:

$$
k=\frac{P}{R \beta_{273.15} R_{273.15}}
$$

其中, $P$ 为热线两端加热功率, 测量相对不确定度小 于 $8 \times 10^{-5} 。 R$ 为热线电阻, 测量相对不确定度小于 $8 \times 10^{-5}$ 。 $\beta_{273.15}$ 为热线在环境温度为 $273.15 \mathrm{~K}$ 下的 电阻温度系数, $R_{273.15}$ 为热线在环境温度为 $273.15 \mathrm{~K}$ 下的热线电阻, 测量相对不确定度小于 $8 \times 10^{-5}$ 。根 据误差传递公式, 测量系统的总热阻 $k$ 的相对不确 定度小于 $10^{-4}$, 结合公式(1), 可以得到测量 $\mathrm{SiC}$ 纤 维热导率的相对不确定度小于 $1 \%$ 。

塞贝克 $\mathrm{T}$ 型法中, 测量交流电压均方根使用 NI-PXI-5922, 其相对不确定度为 $0.06 \%$, 测量赛贝 克电势采用 Keithley 2002, 其相对不确定度小于 $5 \times 10^{-5}$ 。 $\mathrm{SiC}$ 热导率 $\lambda_{\mathrm{f}}$ 使用直流 $\mathrm{T}$ 型法的测量结果, 其相对不确定度小于 $1 \%$, 因此测量 $\mathrm{SiC}$ 赛贝克系数 的相对不确定度小于 $1 \%$ 。

\section{2 结果与讨论}

实验测量了 3 种热处理温度和未进行热处理的 KD-II 型 $\mathrm{SiC}$ 材料的电导率、热导率和塞贝克系数。 热处理在氩气中进行, 热处理温度分别为 $1400^{\circ} \mathrm{C}$ 、 $1500^{\circ} \mathrm{C}$ 和 $1600^{\circ} \mathrm{C}$ 。热处理升温速率为 $300^{\circ} \mathrm{C} / \mathrm{h}$, 达到 设定温度后保持恒温 $1 \mathrm{~h}$ 。热处理的三种样品和未热 处理的样品的尺寸信息如表 1 所示。

\subsection{KD-II 型 SiC 纤维的电导率}

$\mathrm{SiC}$ 纤维的电导率采用综合 $\mathrm{T}$ 型法中的四线制 $\mathrm{T}$ 型法测量。图 2 是环境温度在 80 290 K 范围内, 未进行热处理以及经 $1400 、 1500$ 和 $1600^{\circ} \mathrm{C}$ 热处理 的 $\mathrm{KD}-\mathrm{II}$ 型 $\mathrm{SiC}$ 纤维的电导率。由图 2 可见, 四种 条件下 $\mathrm{SiC}$ 纤维的电导率均随环境温度升高而增大, 但热处理温度对电导率没有影响。文献[12-15]发现 $\mathrm{SiC}$ 纤维在空气中进行热处理时, $\mathrm{SiC}$ 纤维的电导率 会显著增加; 而本研究的 $\mathrm{SiC}$ 纤维在氩气中进行的 热处理, 其电导率和文献报道有很大差别。

表 1 不同温度热处理的 SiC 纤维的尺寸信息

Table 1 Size details of SiC fibers heat-treated at different temperatures

\begin{tabular}{cccc}
\hline $\begin{array}{c}\text { Heat treatment } \\
\text { temperature }\end{array}$ & Diameter/ $\mu \mathrm{m}$ & $\begin{array}{c}\text { Number } \\
\text { of fibers }\end{array}$ & Length $/ \mathrm{mm}$ \\
\hline Unheated & 12.00 & 130 & 5.31 \\
$1400^{\circ} \mathrm{C}$ & 12.00 & 122 & 5.22 \\
$1500^{\circ} \mathrm{C}$ & 12.00 & 152 & 4.83 \\
$1600^{\circ} \mathrm{C}$ & 12.00 & 91 & 5.62 \\
\hline
\end{tabular}




\section{$2.2 \mathrm{KD}-\mathrm{II}$ 型 $\mathrm{SiC}$ 纤维的热导率}

$\mathrm{SiC}$ 纤维的热导率采用综合 $\mathrm{T}$ 型法中的直流 $\mathrm{T}$ 型法测量。图 3 是环境温度在 80 290 K 范围内, 未 进行热处理以及经 $1400 、 1500$ 和 $1600^{\circ} \mathrm{C}$ 热处理的 $\mathrm{KD}-\mathrm{II}$ 型 $\mathrm{SiC}$ 纤维的热导率。由图 3 可见, 4 种条件 下的 $\mathrm{SiC}$ 纤维的热导率均随着环境温度升高而增 大。同时, 热处理后的 $\mathrm{SiC}$ 纤维的热导率随热处理 温度的升高而增大。在环境温度为 $290 \mathrm{~K}$ 时, 未热 处理的 $\mathrm{SiC}$ 纤维的热导率为 $8.0 \mathrm{~W} \cdot \mathrm{m}^{-1} \cdot \mathrm{K}^{-1}$; 经 1400 、 $1500 、 1600^{\circ} \mathrm{C}$ 热处理的 $\mathrm{SiC}$ 纤维的热导率分别为 $8.1 、 9.3$ 和 $11.6 \mathrm{~W} \cdot \mathrm{m}^{-1} \cdot \mathrm{K}^{-1}$, 其中 $1600^{\circ} \mathrm{C}$ 热处理的 $\mathrm{SiC}$ 纤维的热导率比未热处理时的 $\mathrm{SiC}$ 纤维的热导 率提高了 $42 \%$ 。

为了分析 $\mathrm{SiC}$ 纤维热导率随热处理温度显著变 化的原因, 本文分析了平均 $\beta$ - $\mathrm{SiC}$ 尺寸与 $\mathrm{SiC}$ 纤维 热导率之间的关系。Scherrer 公式 ${ }^{[16]}$ 指出, XRD 谱 图中的半峰宽 $B$ 可以表示为 $B=\mathrm{K} \lambda /(L \cos \chi / 2)$, 其中 $\mathrm{K}$ 是一个常数, $\mathrm{K}=2(\ln 2 / \pi)^{1 / 2} \approx 0.93, \lambda$ 是

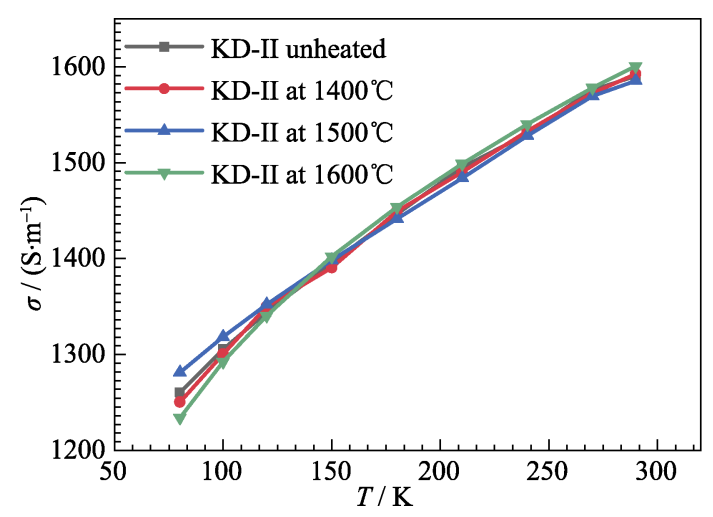

图 $2 \mathrm{SiC}$ 纤维的电导率随环境温度的变化曲线

Fig. 2 Change of electrical conductivity of SiC fibers with environment temperature

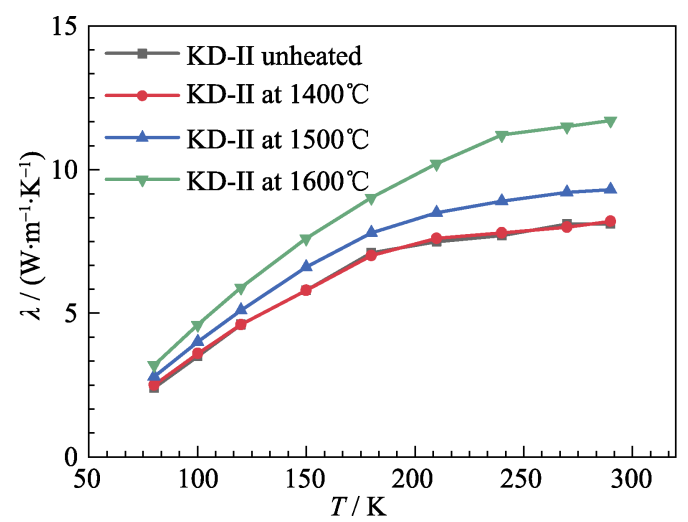

图 $3 \mathrm{SiC}$ 纤维的热导率随环境温度的变化曲线

Fig. 3 Change of thermal conductivity of SiC fibers with environment temperature
$\mathrm{X}$ 射线的波长, $L$ 是晶粒尺寸， $\chi / 2$ 是布拉格角度。 根据 Scherrer 公式, KD-II 型纤维的 $\beta$-SiC 的尺寸可 以根据 XRD 图谱中(111)、(220)和(311)晶面对应的 峰位计算得到。KD-II 型 $\mathrm{SiC}$ 纤维中主要物相为 $\beta$ - $\mathrm{SiC}$, 但当热处理温度超过 $1600{ }^{\circ} \mathrm{C}$ 时, 会出现 $\alpha-\mathrm{SiC}$ 相 ${ }^{[9]}$ 。对比本研究测量的 KD-II 型 $\mathrm{SiC}$ 纤维的 热导率与文献 ${ }^{[17]}$ 中 Tyranno-SA 型 $\mathrm{SiC}$ 纤维的热导 率, 可以发现, $\beta$ - $\mathrm{SiC}$ 平均尺寸每增加 $1 \mathrm{~nm}, \mathrm{SiC}$ 纤维 的热导率平均增加 $1.6 \mathrm{~W} \cdot \mathrm{m}^{-1} \cdot \mathrm{K}^{-1}$, 结果如图 4 所示。

\section{$2.3 \mathrm{KD}-\mathrm{II}$ 型 $\mathrm{SiC}$ 纤维的塞贝克系数}

$\mathrm{SiC}$ 纤维的塞贝克系数采用综合 $\mathrm{T}$ 型法中的交 流加热-直流探测 $\mathrm{T}$ 型法测量。图 5 是环境温度在 80 290 K 范围内, 未进行热处理以及经 1400、1500 和 $1600^{\circ} \mathrm{C}$ 热处理的 $\mathrm{KD}-\mathrm{II}$ 型 $\mathrm{SiC}$ 纤维的塞贝克系 数。当热处理温度在 $1500^{\circ} \mathrm{C}$ 以下时, $\mathrm{SiC}$ 纤维的塞贝 克系数随环境温度和热处理温度的升高而降低。当 热处理温度为 $1600^{\circ} \mathrm{C}$ 时, $\mathrm{SiC}$ 纤维的塞贝克系数几 乎不随环境温度变化。

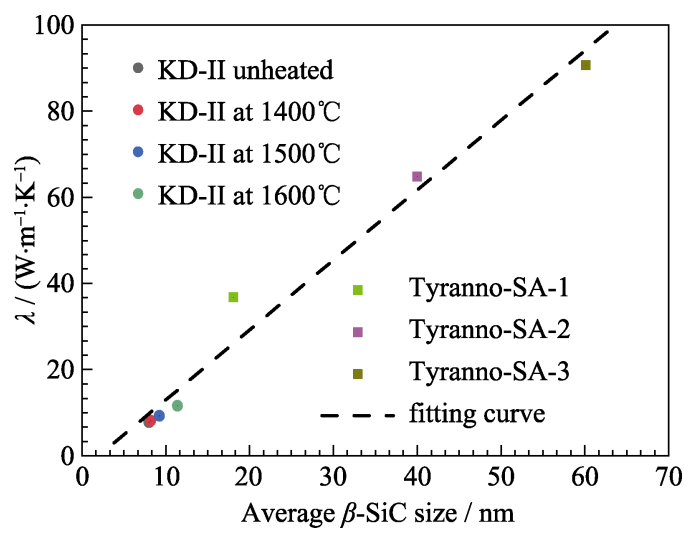

图 $4 \mathrm{SiC}$ 纤维的热导率随平均 $\beta$ - $\mathrm{SiC}$ 尺寸的变化曲线

Fig. 4 Change of thermal conductivity of SiC fibers with size of average $\beta$-SiC

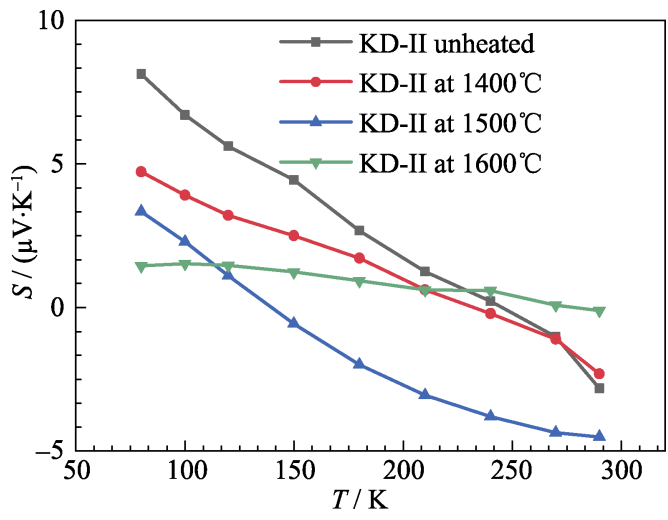

图 $5 \mathrm{SiC}$ 纤维的塞贝克系数随环境温度的变化曲线

Fig. 5 Seebeck coefficient of SiC fibers varies with environment temperature increasing 


\section{3 结论}

实验测量了未经热处理以及经 $1400 、 1500$ 和 $1600^{\circ} \mathrm{C}$ 热处理的 $\mathrm{KD}-\mathrm{II}$ 型 $\mathrm{SiC}$ 纤维的电导率、热导 率和塞贝克系数, 所得结论如下:

1) 4 种条件下的 $\mathrm{SiC}$ 纤维的电导率没有明显变化, 这是由于热处理的氛围为氩气, 没有发生氧化反应。

2) $1400^{\circ} \mathrm{C}$ 热处理的 $\mathrm{SiC}$ 纤维的热导率与未热处 理的 $\mathrm{SiC}$ 纤维的热导率相比, 没有明显变化。当热处 理温度进一步升高时, $\mathrm{SiC}$ 纤维的热导率有显著提高。 当热处理温度为 $1500^{\circ} \mathrm{C}$ 时, $\mathrm{SiC}$ 纤维的热导率比未热 处理的 $\mathrm{SiC}$ 纤维的热导率提高了 $42 \%$, 这是由于升高 热处理温度能够增加 $\mathrm{SiC}$ 纤维中的 $\beta$ - $\mathrm{SiC}$ 的尺寸。

3) 热处理温度在 $1500^{\circ} \mathrm{C}$ 以下时, $\mathrm{SiC}$ 纤维的塞 贝克系数随环境温度和热处理温度的升高而降低; 当热处理温度为 $1600^{\circ} \mathrm{C}$ 时, $\mathrm{SiC}$ 纤维的塞贝克系数几 乎不随环境温度变化。

\section{参考文献:}

[1] WU YI-CAN, WANG WEI-HUA, LIU SONG-LIN, et al. The dual functional lithium lead test blanket module and the testing strategy for ITER. Chinese Journal of Nuclear Science and Engineering, 2005, 25(4): 347-360.

[2] RICCARDI B, GIANCARLI L, HASEGAWA A, et al. Issues and advances in $\mathrm{SiC}_{\mathrm{f}} / \mathrm{SiC}$ composites development for fusion reactors. Journal of Nuclear Materials, 2004, 329: 56-65.

[3] RAFFRAY A, JONES R, AIELLO G, et al. Design and material issues for high performance $\mathrm{SiC}_{\mathrm{f}} / \mathrm{SiC}$-based fusion power cores. Fusion Engineering and Design, 2001, 55(1): 55-95.

[4] JONES R H, GIANCARLI L, HASEGAWA A, et al. Promise and challenges of $\mathrm{SiC}_{\mathrm{f}} / \mathrm{SiC}$ composites for fusion energy applications. Journal of Nuclear Materials, 2002, 307: 1057-1072.

[5] YAJIMA S, HASEGAWA Y, HAYASHI J, et al. Synthesis of continuous silicon carbide fibre with high tensile strength and high young's modulus. Journal of Materials Science, 1978, 13(12): 2569-2576.

[6] BUNSELL A R, PIANT A. A review of the development of three generations of small diameter silicon carbide fibres. Journal of Materials Science, 2006, 41(3): 823-839.

[7] MAH T, HECHT N, MCCULLUM D, et al. Thermal stability of $\mathrm{SiC}$ fibres (nicalon®). Journal of Materials Science, 1984, 19(4): 1191-1201.

[8] YUN H M, DICARLO J A. Comparison of the Tensile, Creep, and Rupture Strength Properties of Stoichiometric SiC Fibers. Proceedings of the 23rd Annual Conference on Composites, Materials and Structures, 1999, 20: 259-72.

[9] CAO S, WANG J, WANG H. Effect of heat treatment on the microstructure and tensile strength of KD-II SiC fibers. Materials Science and Engineering: A, 2016, 673: 55-62.

[10] ZHANG X, FUJIWARA S, FUJII M. Measurements of thermal conductivity and electrical conductivity of a single carbon fiber. International Journal of Thermophysics, 2000, 21(4): 965-980.

[11] MIAO T, MA W, ZHANG X. Ac heating-dc detecting method for seebeck coefficient measurement of the thermoelectric micro/nano devices. Journal of Vacuum Science \& Technology B, Nanotechnology and Microelectronics: Materials, Processing, Measurement, and Phenomena, 2012, 30(5): 051804.

[12] SHIMODA K, COLIN C. Thermo-physical, -mechanical, andelectrical behaviors of ion-irradiated tyranno-sa SiC fibers at high temperatures (1473 K). Journal of Nuclear Materials, 2013, 442(1): S376-S379.

[13] SHIMODA K, COLIN C. Longitudinal dilation behavior of ionirradiated tyranno-sa $\mathrm{SiC}$ fibers at elevated-temperatures. Journal of Nuclear Materials, 2012, 429(1): 298-304.

[14] SMITH C, MORSCHLER G. Determination of silicon carbide fiber electrical resistivity at elevated temperature. International Journal of Applied Ceramic Technology, 2017, 14(2): 228-235.

[15] ZU M, ZOU S M, HAN S, et al. Effects of heat treatment on the microstructures and properties of KD-I SiC fibres. Materials Research Innovations, 2015, 19(sup1): 437-441.

[16] PATERSON A. The scherrer formula for $\mathrm{x}$-ray particle size determination. Physical review, 1939, 56(10): 978.

[17] NAKAYASU T, SATO M, YAMAMURA T, et al. Recent Advancement of Tyranno/SiC Composites r\&d. 23rd Annual Conference on Composites, Advanced Ceramics, Materials, and Structures: B: Ceramic Engineering and Science Proceedings, 1999, 20(4): 301-308. 